(C2007 IEEE. Personal use of this material is permitted. However, permission to reprint/republish this material for advertising or promotional purposes or for creating new collective works for resale or redistribution to servers or lists, or to reuse any copyrighted component of this work in other works must be obtained from the IEEE. 


\title{
POWER SYSTEM SAFETY MANAGEMENT AND AUDIT PROTOCOLS
}

\author{
Muhammad Arshad, Syed M. Islam and Abdul Khaliq
}

\begin{abstract}
The major objective of a safety audit is to determine the effectiveness of the company's safety and loss prevention measures. Identifying potential electrical hazards to prevent or minimize loss of life and property is perceived seriously by the utilities world wide. Safety audit is a methodical approach to evaluate potential hazards enabling suggestions for improvement. It is an important tool for identifying deterioration of standards, areas of risks or vulnerability, hazards and potential incidents in plants for determining necessary action to minimize hazards ensuring effective and meaningful safety efforts. A comprehensive electrical safety audit provides a thorough review of the utility installations, identifies potential electrical hazards, flaws in design system, maintenance system, etc. This paper presents a safety management system and audit protocols to design, plan and execute the electrical safety audit program.
\end{abstract}

\section{INTRODUCTION}

$\mathrm{S}_{\mathrm{s}}$ afety audit is a key element of safety management. Effective management processes are vital to achieve Safety, Health and Environmental higher performance standards. To implement quality safety and loss prevention programs, organization of top management commitment to safety is essential. An electrical safety program is a plan designed so that neither workplace conditions, nor the actions of personnel are exposed unnecessarily to electrical hazards. A well established safety program ensures that people exercise safety procedures effectively. Employers should develop and implement an electrical safety program including safety audit to provide overall safety model. Effective electrical safety audit plays a key role in preventing electrical incidents [1].

Nine fatalities occurred in Ontario, Canada in 2005 due to inadvertent electrocutions. The fatality rate due to electrocution in 2005 was 0.72 deaths per million of population, compared to 0.64 in 2004 . The fatality rate due to electrical fires in 2003 was 0.48 per million of population. The average fatality per annum from 1998 to 2001 was 14 , while the average fatality from 2002 to 2005 was 8.5 [2].

There is a remarkable correlation between quality management and safety. The safety audit also verifies the safety expectations as the product meets quality standards. Safety audits are normally carried out due to various reasons

Manuscript received October 23, 2007.

M. Arshad is with the BC Hydro, Generation Engineering Maintenance Services, Canada (muhammad.arshad@bchydro.bc.ca)

S. Islam, Department of Electrical Engineering and Computer Engineering, Curtin University of Technology, Perth (S.Islam@curtin.edu.au)

A. Khaliq is with the Department of Electrical and Computer Engineering, Islamabad, Pakistan. such as:

1) Regulatory and organizational Occupational Health and Safety (OSH) standards requirements for safety verification.

2) Safety enhancement and standard of performance achievement.

3) Failures/accident involving employee/public safety and or equipment/environmental damage.

4) Near-miss incidents.

5) Plant modification, extension or replacement.

6) New project(s)

The complete safety audit management program should have both the internal and external audit components. The organization should not rely on stand alone internal audit. The internal audit has one of the disadvantages as the avoidance of documenting the hazards due to unwillingness to amend changes. Another disadvantage is that the team members may avoid carrying out critical assessment of a colleague's plant. In addition one may not give importance to a site if nothing serious has happened over a period of time [3]. The internal audit should be designed to investigate, document and present all the weaknesses and strengths to the management including any recommendations. A clear intent and value of the internal audit would have a valuable impact on developing an enhanced safety management system.

The external audit advantages being neutral reporting, and provides an expert analysis and vision of the system from the safety point of view. The external audit identifies hazards that the internal safety might fail to detect. An independent third party audit is worth the expense, and the audit results are certainly justified [4]. The external safety audit team includes the senior manager and subject matter expert. The audit team should be familiar with safety auditing elements, accident investigation, safety training, etc. The audit team should have the ability to interpret rules, standards and to suggest practical cost-effective safety solutions. Safety requirements are expressed in terms of functions and properties of the product for risk assessment. IEC61508 is composed of functional safety assessment based on technical requirement, safety management, and personnel competency. Safety integrity is developed to assess the probability of a safety-related system to satisfactorily perform the required safety functions [5].

Internal audits are recommended every year whereas the external audits are carried out every 2-3 years. It is also recommended that the design review should be carried out 
prior to initiating the safety audit. Records of injury and damage accidents should be examined and used to identify high-risk areas and activities and consequently those which need frequent inspections.

\section{SAFETy AUditing PROCESS}

Up to $80 \%$ of electrical incidents are caused by human errors [6]. An ineffective audit has the potential to undermine carefully nurtured cultural attitudes about quality, standards, procedures and audits [7]. The effectiveness of the safety audit is based on three components such as preaudit preparation and setting protocols, site audit and postaudit analysis including presentation. An effective safety audit program should include elements such as experienced audit team formation, pre-audit briefing, collection and review of relevant information such as past maintenance documentation and accident/failures reports, discussion with plant managers, site visit and outcome/findings presentation to the top management. The scope of audit in compliance to regulatory rules and the vision set by the top management should be set and documented.

The field/plant visit is the most important part of a safety audit program. This involves visiting the plant to identify hazards as per the scope of the audit. All the relevant maintenance documentation and test records are subject to detailed examination. It would be of substantially more value to the auditee for a skilled auditor to discover that, for example, the process for creating and maintaining required documents is missing [7]. Other requirements include:

1) Physical inspection of the facility with reference to applicable standards, rules and codes of practice to identify the strength and weaknesses.

2) Review of adequacy of tools and instruments, including certification.

3) Review of staff credential, test procedures, measurements and analysis in accordance with the applicable valid standards.

An independent safety-assessment (especially when a topdown method is used) shows the proper information which must be collected by an accident investigation as well as during usual system-use, and stored in a data-bank so that an acceptable system-safety monitoring function can be carried out [8].

\section{REPORT FORMAT AND MANAGEMENT BRIEFING:}

Considering aspects such as clarity of the report, usefulness to the client, and to streamline the report, the following format is recommended for the safety audit report:

1) Audit intent and preamble

2) Audit protocols and measurable.

3) Observations, weaknesses and strengths

4) Recommendations and follow-up
The management briefing at the end of safety auditing is an important factor in the effectiveness of auditing because it is the top management who needs to be convinced about the consequences of hazards. A monitoring system is required to ensure that recommendations are communicated and understood that the required work or changes are implemented.

\section{A CASE STUDY}

Fiberglass Reinforced Plastic (FRP) Live line tools high voltage (dielectric) testing service providers' audit was carried out as a part of utility quality safety management system in Canada. The audit team included an external auditor. Six service providers' sites were examined in compliance with regulatory authority and the occupational health and safety standards set by the utility.

\section{A. Audit}

The scope of work included the followings:

1) Review of the written test procedures and followed standards.

2) Test manger and test individuals' interviews.

3) Test personal qualification, training and experience review, i.e. workers performing safety services are qualified and are knowledgeable of the specific tools and equipment they service. "Qualified" means satisfactory in reference to experience, training, education, personal competency, physical fitness, and familiarity with rules, procedures, apparatus and dangers at the work and/or operations.

4) Test equipment calibration and certification. Electrical testing equipment is annually inspected and certified by a Professional Engineer or other approved testing group to confirm the condition of the equipment is acceptable, the characteristics and accuracy of the test voltage are in accordance with testing and maintenance instruction.

5) Inspected electrical testing equipment certification records. Confirm that, within the last 12 months certification has been performed and posted conspicuously by the control panel. Voltage testing equipment and current metering circuits are calibrated to a traceable standard. Performance standards have been compliant for voltage ranges, tripping mechanism in case of fault, crest factor, grounding of equipment, voltage measurement method, interlocks, leakage measurement methods, warning lamps to indicate energized equipment, method of removing corona streamers.

6) Witness the actual test to verify that FRP shafts are correctly high voltage $\mathrm{AC}$ tested. To check the ability of the tester to describe the correct acceptance standard, i.e., leakage current is substantially steady during the one minute test period, and net leakage current does not exceed $50 \mu A$ per $30 \mathrm{~cm}$.

7) Tools repair and maintenance facilities: Confirm that the FRP inspection and assessment procedures include the assessment of physical damage, such as scratches, 
bruising, warping, bending, excessive wear or cracks/separation of glass fibre, identification of dull or dirty surface finishes and, if applicable, moisture/dirt in telescoping sections detection of mechanical flaws, such as cracks, missing teeth, improper action, stripped threads, broken fittings, bent bolts, damaged splines or loose or missing parts.

8) Storage, packing and Shipping: Serviced tools and equipment are protected from accidental damage or contamination from other testing or repair activity. Inspect the areas where tools and equipment are stored after servicing and confirm that adequate protection or separation is provided to prevent contamination from dust and chemical products used in cleaning and finish.

9) Service operation and administration verification to ensure quality control of FRP live line tools which include high voltage dielectric testing test analysis, documentation and communication.

\section{B. Major Findings}

It is essential that the FRP tool (hot-stick) surface be both clean and glossy. The gloss prevents wet-out or sheeting of moisture that can cause conductivity. In this sense, moisture alone is a contaminant. The best procedure is to avoid the use of hot stick in damp or rainy weather. If this is not always possible, the best protection is a clean, glossy tool that will cause water to bead-up, rather than wet-out the surface. It is required to verify hot-stick dielectric properties for the following reasons:

1) Internal conductivity increases due to moisture ingress, dielectric deterioration or internal structural changes.

2) Surface conductivity can result from a combination of loss of gloss, wetting and contamination. Deposits of surface contamination, especially in the presence of moisture, can render even the most perfect tool unsafe.

The followings are the major audit findings:

1) The live line tools, (FRP) dielectric integrity recommended test is $75 \mathrm{kV} / \mathrm{ft}$ ac (at approximately $3 \mathrm{kV}$ per second) to measure the leakage current after one minute [9-11]. It was observed that when $75 \mathrm{kV} / \mathrm{ft}$ is applied the current was not measured per foot, the measured current was the total circuit current divided by the number of per foot sections of the specimen.

2) The current measured is the total current not the leakage current. The $100 \%$ increase in the leakage current would show only $1 \%$ change (increase) in the total current.

3) Calibration certifications required from the legitimate service provider following the traceable standards.

4) No sample of live line tools was tested as delivered to verify its actual status in the field.

5) LS 80 (field test equipment) is considered as good as the service shop high voltage dielectric integrity test. The test due date for LS 80 is two years and for high voltage dielectric integrity test is four years.

6) Test data inventory and documentation electronic version is required.
7) After the refurbishment (finishing/waxing) the tool was tested dry and wet for high voltage dielectric integrity.

\section{Recommendations}

1) Each section (per foot) of the specimen's circuit should have $\mu A$ meter to register the current, the leakage current not the total current.

2) Test equipment should be verified and checked for its calibration at least once in 12 months by the legitimate service provider with traceable standards. The test due stickers must be placed on the equipment. Any calibration (+-\%) should be recorded and considered in the analysis.

3) Proposed $1 \%$ of the tools should be carefully tested for high voltage dielectric integrity as delivered to verify their field status.

4) Refurbished tools should be tested dry and wet for high voltage dielectric integrity before and after waxing is carried out.

5) Electronic database is required to document test data inventory for track record and analysis over a period of time.

6) Study proposal submitted to conclude the effectiveness of LS 80 verses the high voltage dielectric integrity test.

7) Study proposal submitted to conclude quality assurance of all service providers by third party sample test for verification purpose.

8) Study proposal submitted to sample test live line tools (FRP) from various jurisdictions and area of application to determine the test interval accordingly. Collection of samples from different locations will be based on climatic conditions (humidity and salt deposits), usage and quality of storage. Reclamation requires acceptance testing on all new insulating live-line tools, as well as annual inspections and tests for in-service live-line tools. Test required to verify the in service stick performance in actual conditions. The test interval will depend on the outcome of the case study.

Tests are required to be performed to certify quality management and to fulfil the followings.

1) Customer's quality requirements.

2) Applicable regulatory requirements.

3) Aiming to enhance customer satisfaction.

4) Achieve continual improvement of its performance in pursuit of these objectives.

\section{CONCLUSIONS}

A properly designed, planned and executed electrical safety audit program can identify hazards to life and property. An auditor is expected to help the utility to identify the potential electrical hazards to make the utility understand the consequences. An organization instituting safety audits must define the objectives and scope of the audit, its frequency, the elements it should contain and the methods to be used. A well planned audit would provide the followings: 
1) Higher safety standard are met.

2) Maximum practicable operating efficiency is achieved.

3) Premature failures are effectively minimized.

4) Unexpected outages can be minimized ensuring continues power supply to the end user.

5) Overall system reliability is enhanced effectively.

6) Timely relocation/replacement planning is performed with a flexible decision.

7) Life extension is achieved by implementing the enhanced condition based maintenance strategies and restricting the factors responsible for the accelerated aging.

\section{REFERENCES}

[1] Eastwood, K.; Liggett, D.; Hesla, E., "Electrical safety programs," IEEE Transactions on Industry Applications, vol.38, no.6, pp. 16771681, Nov/Dec 2002.

[2] 2005 Ontario (Canada) Electrical Safety Report.

[3] Sreejith, P.G., "Electrical Safety Auditing", IEEE-IEEMA Electrical Safety Workshop on 22nd January 2002, Mumbai, India.

[4] Huddleston, R.L.; Crow, D.R., "A second set of eyes," IEEE Industry Applications Magazine, vol.11, no.3, pp. 63-68, May-June 2005.

[5] May, R., "Safety standards including IEC 61508," Open Control Systems - The Importance of Industrial Standards , vol., no., pp. 6/1615, 26 May 2004.

[6] Inshaw, C.; Wilson, R.A., "Arc flash hazard analysis and mitigation," 58th Annual Conference for Protective Relay Engineers, 2005, vol., no., pp. 145-157, 5-7 April 2005.

[7] Bamford, R.C.; Deibler, W.J., II, "Third-party registrars' audits-for better or for worse?," Third IEEE International Software Engineering Standards Symposium and Forum, 1997. 'Emerging International Standards', ISESS 97, vol., no., pp.164-168, 1-6 Jun 1997.

[8] Vassakis, A.G., "Safety assessment, reliability, and the probabilityoperation diagram," IEEE Transactions on Reliability, vol.45, no.1, pp.90-94, Mar 1996.

[9] IEEE Standard 978-1984, Guide for In-Service Maintenance and Electrical Testing of Live-Line Tools.

[10] ASTM F711-89, Standard Specification for Fiberglass-Reinforced Plastic (FRP) Rod and Tube used in Live-Line Tools.

[11] IEEE recommended practices for safety in high voltage and high power testing, IEEE Std. 510-1983. 